\title{
Psychosocial, Functional, and Emotional Correlates of Long-Term Opioid Use in Patients with Chronic Back Pain: A Cross-Sectional Case-Control Study
}

\author{
Kenta Wakaizumi · Andrew D. Vigotsky • Rami Jabakhanji • \\ Maryam Abdallah · Joana Barroso · Thomas J. Schnitzer • \\ Apkar Vania Apkarian · Marwan N. Baliki
}

Received: December 8, 2020 / Accepted: March 13, 2021 / Published online: April 12, 2021

(C) The Author(s) 2021

\section{ABSTRACT}

Introduction: The opiate epidemic has severe medical and social consequences. Opioids are commonly prescribed in patients with chronic pain, and are a main contributor to the opiate epidemic. The adverse effects of long-term opioid usage have been studied primarily in dependence/addiction disorders, but not in chronic pain. Here, we examine the added

Supplementary Information The online version contains supplementary material available at https:// doi.org/10.1007/s40122-021-00257-w.

K. Wakaizumi · R. Jabakhanji · M. N. Baliki Shirley Ryan AbilityLab, Chicago, IL, USA

K. Wakaizumi · R. Jabakhanji · M. Abdallah · J. Barroso · T. J. Schnitzer · M. N. Baliki ( $₫)$ Department of Physical Medicine and Rehabilitation, Feinberg School of Medicine, Northwestern University, 355 East Erie St, Chicago, IL 60611, USA

e-mail: marwanbaliki2008@u.northwestern.edu

K. Wakaizumi · A. D. Vigotsky · R. Jabakhanji .

M. Abdallah · J. Barroso - T. J. Schnitzer ·

A. V. Apkarian - M. N. Baliki

Center for Translational Pain Research, and Center of Excellence for Chronic Pain and Drug Abuse Research, Northwestern University Feinberg School of Medicine, Chicago, USA

K. Wakaizumi

Department of Anesthesiology, Keio University

School of Medicine, Tokyo, Japan iatrogenic effects, psychology, and brain morphology of long-term opioid use in matched patients with chronic pain with and without opioid use (case-controlled design).

Methods: We compared psychosocial, functional, and psychological measures between patients with chronic back pain (CBP) who were managing their pain with or without opioids, thereby controlling for the effect of pain on these outcomes. In addition, we investigated brain morphological differences associated with longterm opioid usage. We recruited 58 patients with CBP, 29 of them on long-term opioids and 29 who did not use opioids, and who were matched in

\section{A. D. Vigotsky}

Department of Biomedical Engineering,

Northwestern University, Evanston, IL, USA

\section{A. D. Vigotsky}

Department of Statistics, Northwestern University, Evanston, IL, USA

\section{A. V. Apkarian}

Department of Physiology, Northwestern University

Feinberg School of Medicine, Chicago, USA
A. V. Apkarian ( $\square$ )
Department of Anesthesia, Feinberg School of Medicine, Northwestern University, Tarry Bldg. 7-705, Chicago, IL 60611, USA
e-mail: a-apkarian@northwestern.edu 
terms of age, sex, pain intensity, and pain duration. Questionnaires were used to assess pain quality, pain psychology, negative and positive emotions, physical, cognitive, sensory, and motor functions, quality of life, and personality traits.

Results: Patients with CBP on opioids displayed more negative emotion, poorer physical function, and more pain interference $(p<0.001)$, whereas there were no statistical differences in cognitive and motor functions and personality traits. Voxel-based morphometry using structural brain imaging data identified decreased gray matter density of the dorsal paracingulate cortex (family-wise error-corrected $p<0.05$ ) in patients with opioids, which was associated with negative emotion $(p=0.03)$. Finally, a volumetric analysis of hippocampal subfields identified lower volume of the left presubiculum in patients on opioids $(p<0.001)$.

Conclusion: Long-term opioid use in chronic pain is associated with adverse negative emotion and disabilities, as well as decreased gray matter volumes of specific brain regions.

Keywords: Back pain; Opioids; NIH Toolbox; Neuroimaging; Morphometry; Psychology

\section{Key Summary Points}

Why carry out this study?

Long-term opioid usage is still prevalent for managing chronic back pain and a major contributor for the ongoing opioid epidemic.

The psychologic and neurobiological correlates of long-term opioid usage in chronic pain remain unclear and are the primary aim of the current study.

\section{What was learned from the study?}

Long-term opioid use in patients with chronic back pain (in contrast to patients not using opioids) was associated with (1) higher negative emotions and worse disability; (2) decreased gray matter volume of the paracingulate cortex and hippocampus after adjusting for pain intensity and duration.
We evidence adverse psychosocial, functional, and neurological correlates of long-term opioid use in chronic back pain.

\section{DIGITAL FEATURES}

This article is published with digital features, including a summary slide, to facilitate understanding of the article. To view digital features for this article go to https://doi.org/10.6084/ m9.figshare.14207990.

\section{INTRODUCTION}

Chronic pain is defined by the International Association for the Study of Pain as pain that lasts or recurs for longer than 3 months [1]. It affects between $11 \%$ and $40 \%$ of the adult population in the USA, and is associated with restrictions in daily activities, anxiety and depression, poor perceived health, and imposes a significant reduction in quality of life (QOL) $[2,3]$. Opioids have long been used in the treatment of acute and cancer pain, and over the last two decades, there has been a large increase in their prescription for chronic noncancer pain. Each year, more than 160 million prescriptions for opioids are written in the USA [4]. A recent health care utilization study, specifically regarding chronic back pain in the USA where 55,945 patients were assessed, concludes that between the years 2009 to 2016, $66 \%$ of these patients used prescription medications for pain control and the majority (57\%) were consuming opioids [5]. Moreover, the National Center for Health Statistics reports that there is still a consistent increase in drug overdose deaths involving synthetic opioids [6]. Given the ongoing opiate pandemic in the USA, perhaps these numbers have decreased somewhat [7]. Yet, there is little doubt that a large proportion of patients with chronic pain continue on long-term opioids for managing pain. 
The Centers for Disease Control and Prevention (CDC) recommends balancing the benefits and risks of opioid therapy in its Prescribing Opioids for Chronic Pain guidelines [8]. However, there is no consensus about what instruments are needed to assess the benefits and risks. Indeed, there is limited evidence that opioids provide long-term effectiveness in chronic pain, whereas short-term efficacy of opioids is consistently seen in both neuropathic and musculoskeletal pain in two systematic reviews [8]. On the other hand, long-term opioid therapy induces several adverse events including opioid abuse and/or dependence [9, 10]; hyperalgesia [11]; and increased allcause mortality in older people compared with other analgesics [12]. Therefore, quantifying the relationships between long-term opioid use and functional, psychological, and pain properties in patients with chronic pain is seminal for hypothesis-based experiments and, eventually, proper clinical decision-making for treating pain.

Chronic pain is a multidimensional disorder that is related to impaired psychological, emotional, cognitive, and physical function [13-16]. Given that these domains are commonly affected by both pain and opioid treatment, disentangling the influence of the disease and the effects of the drugs is not straightforward: the isolated effect of opioids is difficult to identify without adjusting for the influence of the chronic pain itself. Although opioids have been dispensed to millions of patients with chronic pain, it is surprising how little science has been performed regarding the interaction between opioids and chronic pain. Given recent advances, one can no longer assume that the effects of opioids studied in healthy subjects apply to patients with chronic pain [17]. With this in mind, we conducted a cross-sectional case-control study, in which pain location, intensity, and duration were matched between patients with chronic pain prescribed with and without opioids. Given that pain is a subjective and complex experience that poses a number of measurement challenges, we utilize a compressive set of outcome measures to investigate the effect of long-term opioid use on pain and psychological, emotional, cognitive, and physical properties of patients with chronic pain. We hypothesized that patients on opioids would have poorer psychosocial, functional, and sensorimotor scores relative to patients who do not take opioids.

Secondarily, we examined morphological gray matter differences associated with longterm opioids using structural brain magnetic resonance imaging (MRI). Previous neuroimaging studies on patients using opioids have identified structural abnormalities in brain areas associated with cognitive performance, emotion, and reward processing. In opioid-dependent patients, lower gray matter volumes were reported in multiple cortical and subcortical areas: insula, prefrontal and cingulate cortex, supplementary motor cortex, thalamus, amygdala, and hippocampus [18-22]. These are brain areas that are commonly reported to differ in patients with chronic pain relative to healthy controls [23-25]. Our study design allows us to further advance this topic by identifying morphological brain alterations associated with long-term opioid use in those with chronic pain. We hypothesized that our findings would be in line with previous literature: opioid use would be associated with decreased gray matter in the motor and limbic systems.

\section{METHODS}

\section{Participants}

We recruited patients with chronic low back pain (CBP) lasting for at least 6 months, who were on opioid therapy for at least 3 months (opioid group). Controls were patients with CBP without opioid usage history (non-opioid group), who were matched to the opioid group in terms of age, sex, pain intensity, and pain duration. All participants were older than 18 years, fluent English speakers, and were able to understand instructions as well as questionnaires.

Participants were excluded if they (1) were treated with a spinal cord stimulator; (2) reported rheumatoid arthritis, ankylosing spondylitis, acute vertebral fractures, fibromyalgia, low back/spine oncologic history, 
and other comorbid neurological disorders including major depression and psychiatric disorder requiring treatment; (3) were involved in litigation regarding their back pain, having a disability claim, or receiving workman's compensation; (4) reported a significant other medical disease such as uncontrolled hypertension, unstable diabetes mellitus, renal insufficiency, congestive heart failure, coronary or peripheral vascular disease, chronic obstructive lung disease, or malignancy; (5) were pregnant during the study.

Overall, 29 patients with CBP with opioid (opioid group) and 29 without opioid (nonopioid group) were included in this study. Power analysis had shown that 25 individuals in each group were able to detect an effect size of 1.5 between two groups with statistical power 0.8 , assuming alpha was 0.01 (two-tailed). This study was performed in accordance with the Helsinki Declaration of 1964 and its later amendments. All participants understood the purpose of the study, procedures to be completed, possible benefits and potential risks, and signed a consent form prior to any study-related activities. The protocols were approved by Northwestern University IRB (STU00207384, STU00205398).

\section{Psychosocial, Sensorimotor, and Opioid Measures}

All participants completed a demographic survey asking about their age, gender, smoking status, height, weight, history of surgery, history of back surgery, pain intensity, and pain duration. Pain intensity was measured by the Numerical Rating Scale (NRS) [26]. Opioid users reported details of prescribed opioids and the Current Opioid Misuse Measure (COMM), which estimates a risk of opioid misuse. Dosage of prescribed opioids was converted to the morphine milligram equivalent (MME) using standard conversion factors generated by the CDC [27].

Multidimensional assessment for patients consisted of 64 measures in 10 distinct instruments: (1) Oswestry Disability Index (ODI) [28], (2) PainDETECT [29], (3) McGill Pain
Questionnaire-short form (MPQ) [30], (4) Pain Catastrophizing Scale (PCS) [31], (5) Beck Depression Inventory (BDI) [32], (6) Positive and Negative Affect Schedule (PANAS) [33], (7) Patient-Reported Outcomes Measurement Information System (PROMIS) profile test [34], (8) 12-item Short Form (SF12) health survey [35], (9) Multidimensional Assessment of Interoceptive Awareness (MAIA) [36], and (10) NIH Toolbox [37] (containing four domains of sensory [38-40], emotion [41], cognition [42], and motor function[43]). All questionnaires were collected electronically using REDCap, a browser-based software and workflow methodology for designing clinical and translational research databases. Details of all collected measures are listed below.

NRS

COMM A self-reported questionnaire containing 17 items, which estimate the risk of opioid misuse [44].

ODI A ten-item questionnaire, measuring functional disability in daily living [28].

PainDETECT A seven-item screening questionnaire for neuropathic pain [29].

MPQ A 15-item measure of affective and sensory components of pain [30].

PCS A 13-item assessment for thoughts or feelings related to past pain experiences, yielding three subscale scores assessing rumination, magnification, and helplessness [31].

BDI A 21-item instrument to measure the severity of depression [32].

PANAS A composite questionnaire containing two mood scales rated on ten items in each scale; one measures negative 
affect and the other measures positive affect [33].

PROMIS This profile test contains 57 items for seven core domains of health-related quality of life (QOL); pain interference, fatigue, depression sadness, anxiety fear, sleep disturbance, physical function, and social activity [34].

SF12 A composite questionnaire of eight sections measuring QOL; general health, physical role function, physical function, social function, emotional role function, mental health, vitality, and bodily pain [35].

MAIA A 32-item questionnaire measuring seven personal traits of the interoceptive awareness; not distracting, not worrying, noticing, attention regulation, emotional regulation, selfregulation, body listening, and trusting [36].

NIH Toolbox In this study, the sensory part of the NIH Toolbox is composed of pain interference [38], regional taste quinine and salt [39], and odor identification [40]. Emotional assessment of the NIH Toolbox contains ten measures of negative emotion: anger-physical aggression, anger-affect, anger-hostility, sadness, fear-affect, fearsomatic arousal, perceived hostility, perceived stress, perceived rejection, and loneliness; and seven measures of positive emotion: general life satisfaction, meaning and purpose, positive affect, friendship, emotional support, self-efficacy, and instrumental support [41]. The NIH Toolbox also assesses six cognitive functions: picture vocabulary, picture sequence memory, flanker inhibitory control and attention, dimensional change card sort, pattern comparison processing speed, list sorting working memory [42]. The 2-min walk endurance and nine-hole pegboard dexterity dominant and non-dominant were assessed as motor functions of the NIH Toolbox [43] in this study.

\section{Statistical Analyses of Psychosocial and Sensorimotor Measures}

Demographic measures were compared between opioid and non-opioid group using two-tailed unpaired $t$ tests for continuous data or chisquare tests for binary data. A log transformation was used for a comparison of pain duration to approximate to a normal distribution. In case of missing within-questionnaire items, these were replaced with the average of the remaining within-questionnaire scores, provided that the number of unanswered questions was less than $30 \%$ of all items in each scale [45]. If more than $30 \%$ of the items were blank, subjects were excluded from statistical tests relevant to the given scale. In our dataset, because an individual in the opioid group did not answer a large portion of the PROMIS, the individual was excluded from any statistical tests relevant to the PROMIS.

Differences of all 64 measures in the multidimensional assessment between the groups were assessed using a multivariable regression analysis, which adjusted for age, sex, pain intensity, log-transformed pain duration, and history of back surgery. Next, Pearson's correlation of each measure to pain intensity was analyzed in each group. Then, we investigated group difference of the correlation coefficient, where Fisher's $z$ transformation was performed on the correlation coefficients and statistical significance was examined under standard normal distribution. We employed two different alpha thresholds for statistical significance in a multiple comparison manner. The first was a Bonferroni's corrected $p$ value in a small group, in which the thresholds depended on the 
number of measures in each small group. For example, because the PROMIS contains seven measures, the alpha was $0.05 / 7(\approx 0.007)$. Another threshold was a Bonferroni's corrected $p$ value for the entire assessment of 64 measures $(0.05 / 64 \approx 0.0008)$. Effect sizes of the difference between the two means of the two groups were assessed using Cohen's $d$ in MATLAB 2016a (The MathWorks, Natick) and JMP Pro version 13.2 (SAS Institute, Cary, NC).

To reduce the dimensionality of the assessments, a principal component analysis using a varimax rotation was performed on the statistically significant measures identified at the Bonferroni's corrected $p$ value $(p<0.0008)$ in each group. Two criteria for component selection were used: (1) an eigenvalue of more than 2 , and (2) the percentage of explained variance above $10 \%$. After the principal components were selected, component scores corresponding to each patient with CBP were created on each principal component and standardized along subject column to reflect a $Z$ score.

Associations of the identified principal components with pain intensity and opioid-related measures (MME and COMM) were examined using a multivariable regression analysis, adjusted for age, sex, log-transformed pain duration, and history of back pain in each group. In the analysis, log-transformation was performed on the MME to approximate to a normal distribution.

\section{Blood Concentration of Opioids}

Blood samples were drawn for each participant before the MRI scan and psychosocial and sensorimotor assessments. Plasma concentrations of opioids were measured using high-performance liquid chromatography tandem mass spectrometry (HPLC-MS/MS), with the mass spectrometer operating in positive ion and multiple reaction monitoring acquisition mode, after sample preparation by solid-phase extraction or simplified liquid extraction. All samples were run in duplicate and the average concentration used.

The opioid concentrations were then converted to morphine equivalent units (parenteral morphine) using similar method described above [27], and were log-transformed and compared to pain intensity and the identified principal components. Five individuals were excluded from this analysis as the blood sample volumes collected were insufficient.

\section{Scanning Parameters of Magnetic Resonance Imaging}

Participants were scanned on a 3-T Siemens Prisma. High-resolution T1-weighted brain images were acquired using integrated parallel imaging techniques (PAT; GRAPPA) representing receiver coil-based data acceleration methods. The acquisition parameters were isometric voxel size $=1 \times 1 \times 1 \mathrm{~mm}, \quad \mathrm{TR}=2300 \mathrm{~ms}$, $\mathrm{TE}=2.40 \mathrm{~ms}$, flip angle $=9^{\circ}$, acceleration factor of 2 , base resolution 256 , slices $=176$, and field of view $(\mathrm{FoV})=256 \mathrm{~mm}$. The encoding directions were from anterior to posterior, and a time of acquisition of $5 \mathrm{~min} 21 \mathrm{~s}$.

\section{Voxel-Based Morphometry of Cortical Gray Matter}

Intracranial volume normalized for individual head size was estimated with SIENAX [46], part of FSL [47]. Gray matter density was examined using voxel-based morphometry from FSL-VBM [48], an optimized voxel-based morphometry (VBM) analysis [49] in FSL [47]. Statistically significant voxel clusters were identified using threshold-free cluster enhancement (TFCE) [50] with family-wise error corrected $p$ value less than 0.05. Neurosynth term-based reverse inference [51,52] was then used to decode the identified brain region (see details in the supplementary methods).

Gray matter density in the identified cluster was averaged for each participant, then compared between the opioid and non-opioid groups using a multivariable regression analysis which adjusted for age, sex, pain intensity, logtransformed pain duration, history of back surgery, and intracranial volume. Next, we examined the associations of the averaged gray matter density with pain intensity, the identified principal components, log-transformed 
MME, or COMM, again adjusting for age, sex, and intracranial volume. We also performed volumetric analyses of subcortical nuclei and hippocampal subfields (see supplementary material).

\section{Neurosynth Term-Based Reverse Inference}

This meta-analytical tool contains a database with activation coordinates for a total of 14,371 functional MRI studies, paired with their associated cognitive and anatomical terms (http:// neurosynth.org/decode/) $[51,52]$. The decoder takes in the voxel-wise representation of the region, cross-references it with the full database, and returns a list of terms and their correlation values to the region. Here, we retrieved the top 50 non-anatomical terms (of about 1700 terms generated) showing the greatest correlation.

\section{Volumetric Analysis of Subcortical Nuclei}

Volume extraction of subcortical nuclei from T1-weighted images was performed through FSL's integrated registration and segmentation tool (FIRST) [53], which computed volumes of all subcortical nuclei including thalamus, caudate, putamen, pallidum, hippocampus, amygdala, and accumbens. Volume comparison between the groups was performed with a multivariable regression analysis adjusting for age, sex, pain intensity, log-transformed pain duration, history of back surgery, and intracranial volume.

\section{Volumetric Analysis of Hippocampal Subfields}

Volumetric segmentation of hippocampal subfields was performed with FreeSurfer 6.0 [54], which is documented and freely available for download online (http://surfer.nmr.mgh. harvard.edu/), using T1-weighted images. Based on a statistical atlas built primarily upon ultra-high resolution (ca. $0.1 \mathrm{~mm}$ isotropic) ex vivo MRI data [54], this tool generates an automated segmentation of the 24 hippocampal subfields, including left/right parasubiculum, presubiculum, subiculum, cornu ammonis 1 (CA1), CA3, CA4, granule cell and molecular layer of the dentate gyrus (GC-MLDG), molecular layer, hippocampal amygdala transition area (HATA), fimbria, hippocampal tail, and hippocampal fissure. Group differences of these volumes were examined using a multivariable regression analysis adjusting for age, sex, pain intensity, log-transformed pain duration, history of back surgery, and intracranial volume. Significant difference was identified by FEW-p $(0.05 / 24 \approx 0.002)$.

\section{Software}

Statistical analyses were performed using MATLAB 2016a (The MathWorks, Natick) and JMP Pro version 13.2 (SAS Institute, Cary, NC). Brain schema was visualized on a surface rendering of a human brain atlas with the BrainNet Viewer (http://www.nitrc.org/projects/bnv/) [55]. The term visualization was performed using a word cloud (Text Analytical Toolbox in MATLAB).

\section{RESULTS}

Several opioids had been prescribed for patients with CBP in the opioid group: hydrocodone, tramadol, oxycodone, fentanyl, morphine, codeine, oxymorphone, hydromorphone, buprenorphine, and methadone. All patients maintained their current opioid prescriptions at least for 1 year (range of duration of prescription 1-16 years). Median of their MME was $30 \mathrm{mg} /$ day (range $5-1320 \mathrm{mg} /$ day). A greater proportion of the opioid group had a history of back surgery compared to the non-opioid group $(p<0.001)$; however, there were no statistically significant differences in pain intensity, pain duration, age, sex, smoking status, body mass index, or other past surgeries (Table 1).

All participants were assessed multidimensionally with a total of 64 measures, combined in 10 distinct instruments. A patient in the opioid group was excluded from the below statistical analyses relevant to the PROMIS as a result of missing data (see detail of the criteria in the supplementary methods). Bonferroni's corrected threshold for the 64 measures 
Table 1 Demographic characteristics and prescribed opioids in patients with chronic back pain with and without opioids

\begin{tabular}{|c|c|c|c|c|c|c|c|}
\hline \multirow[b]{2}{*}{ Age (years), mean (SD) } & \multicolumn{2}{|c|}{ Non-opioid $(n=29)$} & \multicolumn{2}{|c|}{ Opioid $(n=29)$} & \multirow{2}{*}{$\frac{t \text { or } \chi^{2}}{0.30}$} & \multirow{2}{*}{$\frac{p \text { value }}{0.76}$} & \multirow{2}{*}{$\frac{\text { Effect size }}{0.04}$} \\
\hline & 57.7 & $(12.5)$ & 58.6 & $(10.0)$ & & & \\
\hline Male, $n(\%)$ & 13 & $(44.8)$ & 13 & $(44.8)$ & 0.00 & 1.00 & 0 \\
\hline Current smoker, $n(\%)$ & 5 & $(17.2)$ & 10 & $(34.5)$ & 2.28 & 0.13 & 0.20 \\
\hline BMI $\left(\mathrm{kg} / \mathrm{m}^{2}\right)$, mean $(\mathrm{SD})$ & 30.0 & $(6.4)$ & 29.9 & $(6.9)$ & -0.08 & 0.94 & -0.01 \\
\hline History of surgery, $n(\%)$ & 21 & $(72.4)$ & 25 & $(86.2)$ & 1.71 & 0.19 & 0.17 \\
\hline History of back surgery, $n(\%)$ & 1 & $(3.5)$ & 11 & $(37.9)$ & 11.94 & $<0.001$ & 0.45 \\
\hline Pain intensity, mean (SD) & 6.8 & $(1.5)$ & 6.1 & $(1.9)$ & -1.38 & 0.17 & -0.25 \\
\hline Pain duration ${ }^{\#}$ (years), median (min, max) & 12 & $(0.9,50.0)$ & 17 & $(2.0,54.3)$ & 1.79 & 0.08 & 0.23 \\
\hline \multicolumn{8}{|l|}{ Prescribed opioids } \\
\hline Hydrocodone, $n$ (\%) & \multicolumn{2}{|l|}{-} & 11 & $(37.9)$ & - & - & \\
\hline Tramadol, $n(\%)$ & \multicolumn{2}{|l|}{-} & 7 & $(24.1)$ & - & - & \\
\hline Oxycodone, $n$ (\%) & \multicolumn{2}{|l|}{-} & 6 & $(20.7)$ & - & - & \\
\hline Fentanyl, $n(\%)$ & \multicolumn{2}{|l|}{-} & 2 & (6.9) & - & - & \\
\hline Morphine, $n(\%)$ & \multicolumn{2}{|l|}{-} & 2 & $(6.9)$ & - & - & \\
\hline Codeine, $n(\%)$ & \multicolumn{2}{|l|}{-} & 2 & (6.9) & - & - & \\
\hline Oxymorphone, $n(\%)$ & \multicolumn{2}{|l|}{-} & 1 & $(3.4)$ & - & - & \\
\hline Hydromorphone, $n$ (\%) & \multicolumn{2}{|l|}{-} & 1 & $(3.4)$ & - & - & \\
\hline Buprenorphine, $n(\%)$ & \multicolumn{2}{|l|}{-} & 1 & $(3.4)$ & - & - & \\
\hline Methadone, $n(\%)$ & \multicolumn{2}{|l|}{-} & 1 & $(3.4)$ & - & - & \\
\hline \multicolumn{3}{|c|}{ Morphine milligram equivalent (mg/day), median (min, max) } & 30 & $(5,1320)$ & - & - & \\
\hline \multicolumn{3}{|c|}{ Current Opioid Misuse Measure (COMM), mean (SD) } & 10.5 & $(7.6)$ & - & - & \\
\hline
\end{tabular}

Two-tailed unpaired $t$ test or chi-square test were performed. Effect sizes are presented as Pearson's $r$ for continuous outcomes and $r_{\varphi}$ for categorical outcomes; thus, both are interpretable on the correlation coefficient scale

$B M I$ body mass index, $S D$ standard deviation

\# Pain duration was log-transformed for statistical analysis

$(p=0.0008)$ identified statistical significance in 20 measures, including ODI, rumination, magnification, and helplessness in the PCS, BDI, negative affect in the PANAS, pain interference, fatigue, depression-sadness, anxiety-fear, physical function, and social activity in the PROMIS, pain interference, sadness, and perceived stress in the NIH Toolbox, and general health, physical role function, physical function, social function, and bodily pain in the SF12 (Fig. 1,
Table S1 in the supplementary material). However, there were no statistically significant differences across the cognitive and motor measures in the NIH Toolbox and the seven personal traits measured in the MAIA. In addition, pain intensity was significantly associated with anxiety-fear in the PROMIS in the opioid group, and the correlation coefficient ( $r$ value) significantly differed from one in the non-opioid group (Fig. 1, Table S2 in the supplementary 
material). Bonferroni's corrected threshold in the PROMIS $(p<0.007)$ identified that depression-sadness was significantly associated with pain intensity in the opioid group and its $r$ value statistically differed from the non-opioid group. This result was consistent in a subsample without experience of back surgery $(n=46$, statistical details not shown).

To further characterize these domains and their potential relationship with brain properties, principal component analysis was performed on the 20 identified measures, reducing dimensionality of the assessments. This resulted in three principal components (PCs) in the opioid group, labeled according to the measures' loadings (Fig. 2, Table S3 in the supplementary material): negative affect, physical function, and pain interference (eigenvalues were $7.83,2.93$, and 2.09 , respectively). On the other hand, we identified two PCs, pain interference and negative affect, in the non-opioid group, because physical function was not segregated from the component of pain interference (Table S4 in the supplementary material). Multivariable regression analyses identified that negative affect and physical function were associated with pain intensity $(p<0.001$ and $p=0.02$, respectively), although neither MME nor COMM showed statistically significant associations with the PCs in the opioid group (Table 2). In contrast, pain intensity was not statistically associated with pain interference or negative affect in the non-opioid group (Table S5 in the supplementary material). Despite having a modest correlation with the MME of prescribed opioids $(r=0.48 ; 95 \%$ CI 0.11-0.74), blood concentration of opioids showed negligible associations with pain intensity, negative affect, physical function, or pain interference ( $r=-0.21$ to 0.13 ; Fig. S1 in the supplementary material).

Brain gray matter morphological properties were contrasted between patients with CBP with and without opioids using T1-weighted MRI. First, we applied a whole brain VBM analysis, which identified a cluster with lower gray matter density in the dorsal paracingulate cortex (dPaCC) in the opioid group (Fig. 3a). The Neurosynth meta-analytical decoding tool was utilized to show the top 50 terms with the greatest correlation to the cluster. As shown in a word cloud, the top five terms were evaluations, inferences, beliefs, social, and choice (Fig. 3b). Furthermore, decreased gray matter density in the dPaCC was significantly associated with increased pain intensity $(p=0.005)$ and negative affect $(p=0.017)$, but not with physical function or pain interference in patients with CBP with opioid (Table S6 in the supplementary material), whereas the gray matter density was not statistically associated with pain intensity nor negative affect in patients without opioid (Fig. 3c). Neither MME nor COMM was statistically significantly associated with the gray matter density in this cluster (statistical details were not shown). Lastly, a volumetric analysis of hippocampal subfields identified statistically significant smaller volume of the left presubiculum in patients with CBP with opioid compared to those without opioid (Bonferroni's collected $p$ value $<0.05$, Table $S 8$ in the supplementary material). However, the volume was not statistically associated with pain intensity, identified principal components, MME, or COMM (statistical details not shown).

\section{DISCUSSION}

In this study, we investigated correlates of longterm opioid use, adjusted for pain intensity and duration, in patients with CBP treated with and without opioids. Negative affect-including depression, anxiety, fear, sadness, and perceived stress-was consistently worse in the opioid group, over several instruments such as BDI, PANAS, PROMIS, and NIH Toolbox. The interaction of these domains and opioid therapy has been studied previously: a cross-over drug administration study found that pain catastrophizing and negative affect decrease the effect of endogenous opioid and external opioid response in patients with CBP [56]. In addition, these psychological factors are considered to attenuate the conditioned pain modulation, which is a phenomenon responsible for a centrally mediated analgesic response [57]. Furthermore, a large epidemiological study found that long-term opioid use itself induces depression [58]. Opioid drugs are also implicated with 
A

Pain characteristics

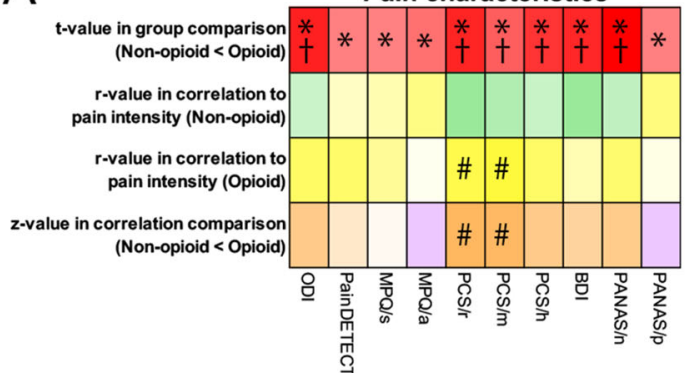

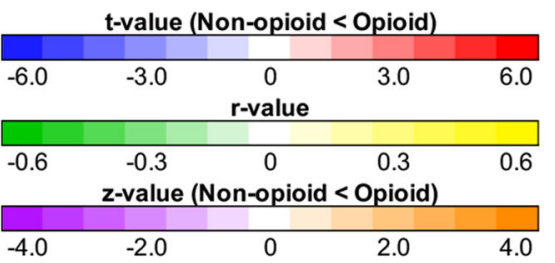
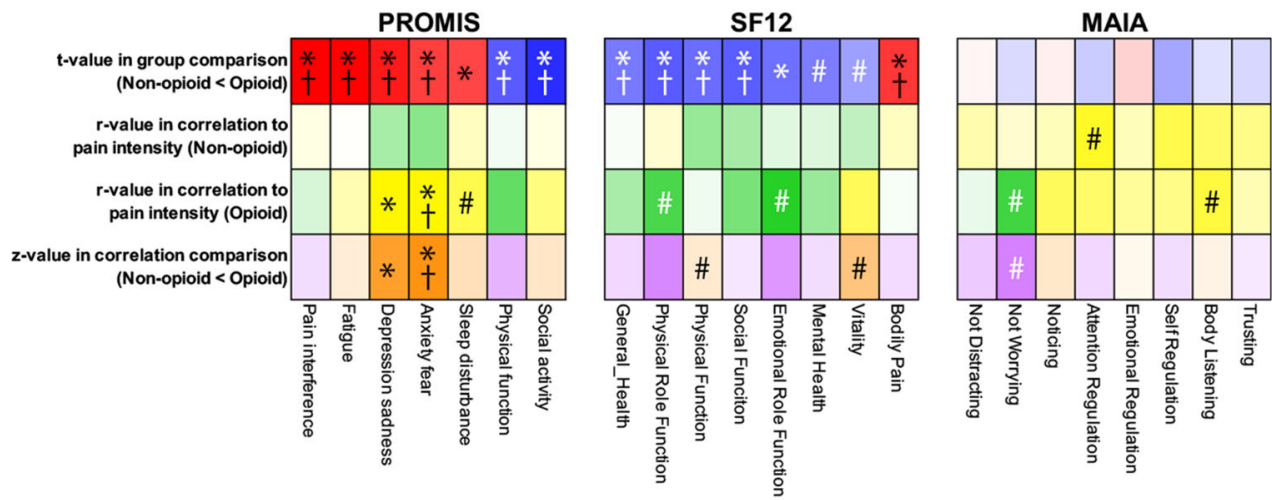

NIH toolbox

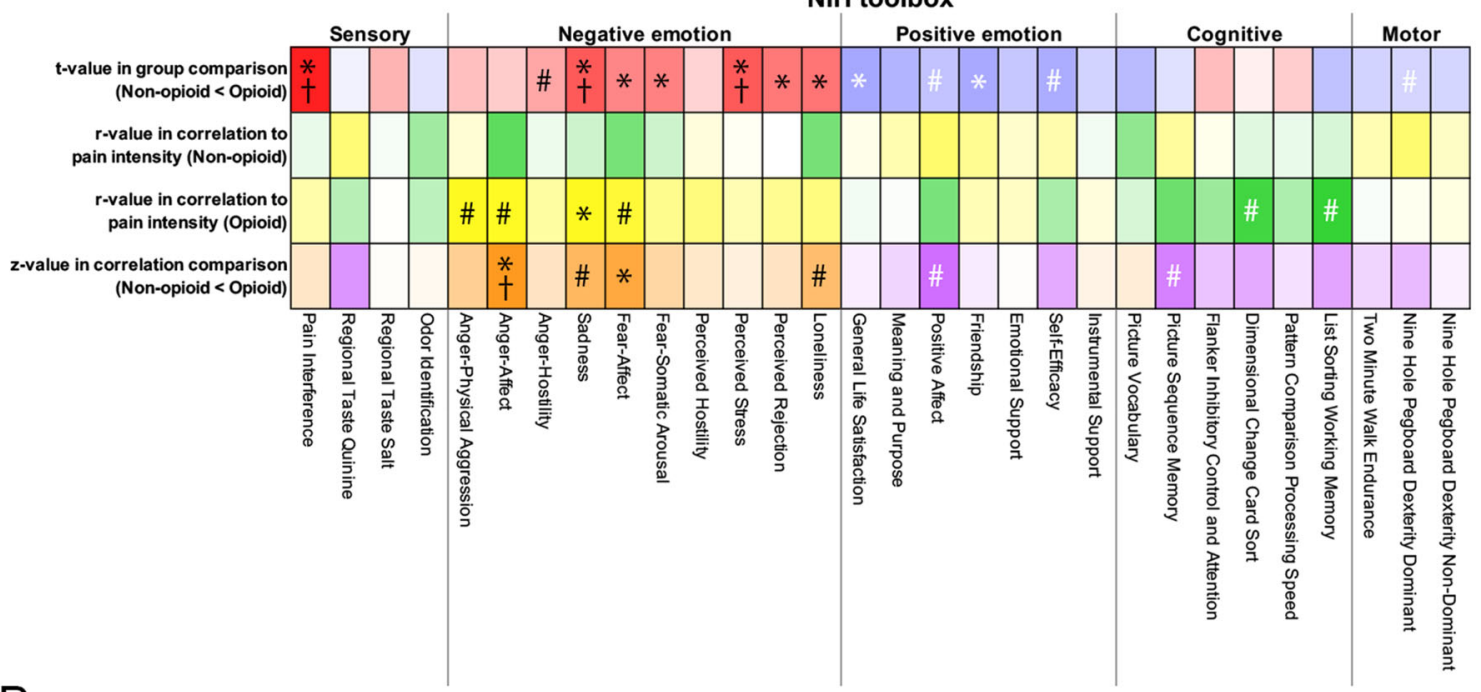

B

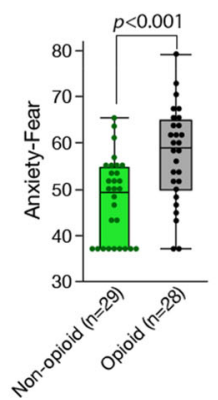

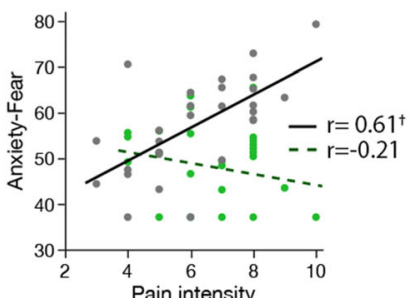

- Non-opioid $(n=29)$ - Opioid $(n=28)$

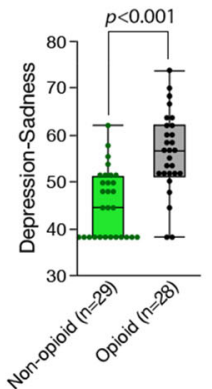

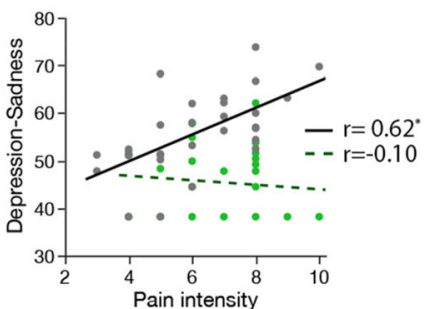

- Non-opioid ( $n=29) \quad-$ Opioid $(n=28)$ 
4Fig. 1 Group comparisons of multidimensional measures between patients with chronic back pain on long-term opioid use $(n=29)$ and those without opioid use $(n=29)$. a Heat maps of statistical values in multidimensional measures. The first row represents $t$ values of group effect (non-opioid < opioid groups) in multivariable regression analyses adjusted for age, sex, pain intensity, pain duration, and history of back surgery. The second and third rows show correlation coefficients ( $r$ values) of pain intensity to the measures in the non-opioid and in the opioid groups, respectively. The fourth row represents $z$ values in the correlation comparison (non-opioid $<$ opioid groups). b Group differences of anxiety-fear and depression-sadness in the PROMIS and their correlations to pain intensity. Box plot shows the upper and lower quartiles and median, and whiskers represent the highest and lowest values. Scatter plots show correlations of pain intensity. Solid and dot lines in the scatter plots show fitted lines in opioid and non-opioid groups, respectively. A patient in the opioid group was excluded from the statistical tests regarding the PROMIS as a result of missing data. ODI Oswestry Disability Index, MPQ McGill Pain Questionnaire, MPQ/a MPQ/affective component, $\mathrm{MPQ} / \mathrm{s} \mathrm{MPQ} /$ sensory component, PCS Pain Catastrophizing Scale, PCS/h PCS/helplessness, PCS $/ \mathrm{m}$ $\mathrm{PCS} /$ magnification, PCS/r PCS/rumination, PANAS Positive And Negative Affect Scale, BDI Beck Depression Inventory, PANAS/n PANAS/negative affect, PANAS/p PANAS/positive affect. ${ }^{\#}$ Uncorrected $p$ value $<0.05$, ${ }^{*} p$ value less than a Bonferroni's corrected threshold in each small group of the measures (exact thresholds depend on number of measures in each small group), ${ }^{\dagger} p$ value less than a Bonferroni's corrected threshold in whole measures $(0.05 / 64 \approx 0.0008)$

dopaminergic reward system disturbance, by increasing dopamine expression through opioid processing neurons. Long-term exposure to opioids results in a reduced number of dopamine receptors in a regulatory negative feedback loop [59]. Reduced dopamine receptors are also associated with anhedonia, or a loss of pleasure [60]. Thus, worse negative affect may be linked to dysfunction of the brain reward system associated with long-term opioid use.

In this study, the negative affect was associated with pain intensity in patients with opioids (standardized beta $=0.81$; Table 2 ), indicating that the dysfunction of the reward system may be a critical mechanism of severe chronic pain in patients treated with long-term opioid. Given that opioid use is associated with opioid tolerance [61] and opioid-induced hyperalgesia [11], adverse negative affect is probably an indicator of these paradoxical opioid dysfunctions induced by the reward system disorder for patients with chronic, refractory pain during long-term opioid therapy. Opioid tapering program and alternative multidisciplinary pain management [62] need to be taken into consideration for those patients.

Impairments in reported physical function and pain interference in the opioid group were consistent across multiple scales (ODI, PROMIS, NIH Toolbox, and SF12), whereas there were no statistically significant differences on motor, cognitive, or sensory (taste and smell) functions in the NIH Toolbox. That is, long-term opioid use is associated with differences in patient-reported outcomes but not necessarily fundamental motor, cognitive, and sensory functions in patients with CBP. Although negative affect is associated with physical disability [63, 64], the principal component analysis on statistically significant discriminative tests for opioidtreated patients identified physical function and pain interference as distinct components. Items contained in the instruments indicate that the second component, physical function, assesses personal activities of daily living, whereas the third component, pain interference, is more related to social activities. These findings suggest that long-term opioid use is associated with both personal and social disabilities, which are independent properties from chronic pain and negative affect. The opioidassociated patient-reported disability is consistent with a previous prospective cohort study, which found that prescribed opioids increased self-rated physical disability at the 6-month follow-up in patients with low back pain, even after adjusting for confounders, using propensity scores reflecting the probability of opioid prescription given baseline characteristics [65].

In individuals with CBP treated with opioids, we identified lower gray matter density in the $\mathrm{dPaCC}$, which is considered to represent a cingulofrontal transition zone between the anterior cingulate cortex (ACC) and adjacent prefrontal regions [66]. As the Neurosynth 
A

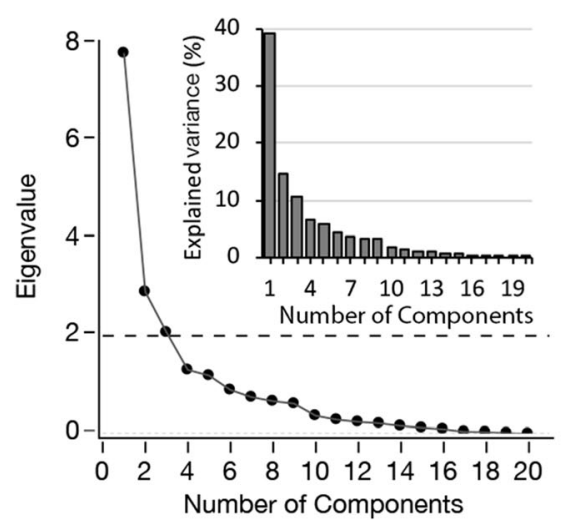

Fig. 2 Principal component analysis of psychosocial and sensorimotor measures showed significant group differences $(n=28)$. a A scree plot of eigenvalues and a bar graph of explained variances. Principal components were identified by criteria of (1) eigenvalue $\geq 2.0$ and (2)

decoder identified, functional MRI studies reveal that $\mathrm{APaCC}$ is involved in reward-based decision-making $[67,68]$. Furthermore, positron emission tomography (PET) has evidenced a rich density of mu-opioid receptors in the PaCC and the ACC [69]. Therefore, gray matter volume in the dPaCC may be affected by opioid administration [70]. In the present study, decreased gray matter density of the $\mathrm{dPaCC}$ was associated with negative affect in patients with CBP with opioids. This finding is consistent with previous studies, which have demonstrated decreased gray matter volume of the PaCC/ACC in people with depression [71, 72]. Although chronic pain probably generates a similar morphological change [73], our findings, which control for pain properties, suggest the long-term opioid use is associated with decreased gray matter volume of the dPaCC, in association with increased negative affect in patients with CBP. A meta-analysis found reduced gray matter volume in the frontotemporal regions, including the medial prefrontal cortex and the insula cortex, in opioid-dependent patients [74]; these regions were not identified in this study. Given our result, and the fact that frontotemporal regions are also commonly altered by chronic pain, we believe
Negative affect Physical function Pain interference identified three principal components: negative affect, physical function, and pain intensity

these changes may not be specific to the opioid use [25].

In the present study, there were no statistically significant psychosocial or neurological associations with the dosage of prescribed opioids, with the opioid misuse scale, or with the blood concentration of opioids. Two cross-sectional studies have suggested that psychological distress including high pain catastrophizing, depression, and anxiety are predictors of opioid misuse in patients with chronic pain $[75,76]$. Since this study had small number of subjects, statistical power may not have been sufficient to identify the psychosocial associations with a risk of opioid misuse. In addition, this study included patients using several types of opioids, i.e., immediate-release and extended-release or long-acting (ER/LA) opioids. ER/LA opioids, such as buprenorphine and methadone, are sometimes prescribed to reduce opioid addictions and abuses [77, 78], intimating that patients may have different psychosocial and sensorimotor properties corresponding to distinct opioid prescriptions. Opioid tolerance often occurs during long-term opioid use [61], suggesting that the amount of opioid intake or blood concentration does not reflect a fixed effect across patients. Even though the dose of opioids was converted to the equivalent one of 


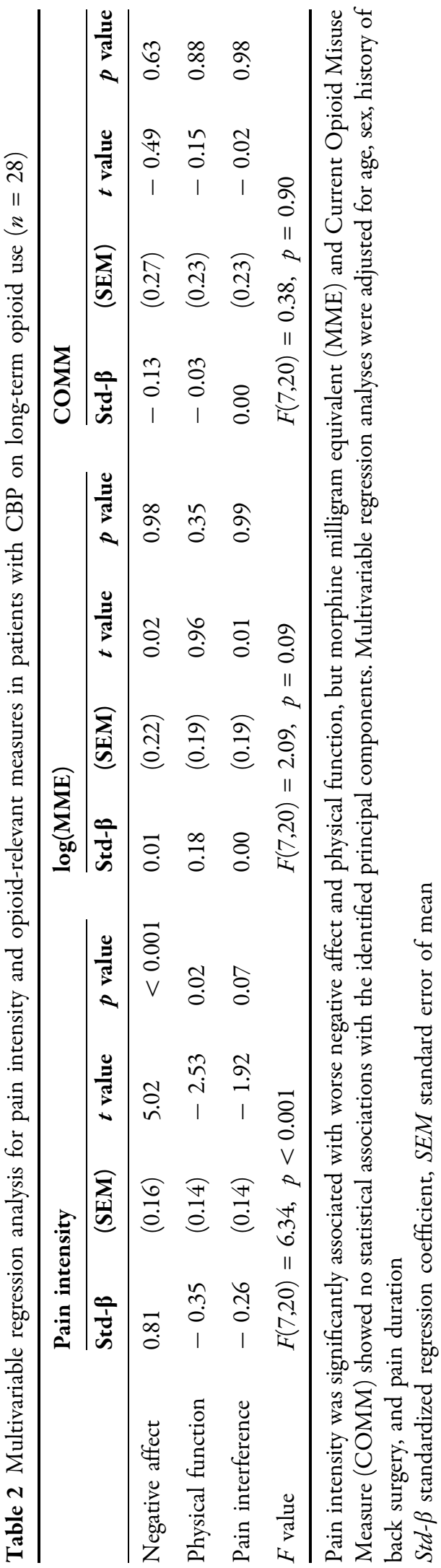

morphine using standard conversion factors generated by the CDC [27], it was not surprising that no psychosocial and neurological measures were statistically significantly associated with the opioid dosages.

There are several limitations in this study. Firstly, our findings come from of a cross-sectional study design, in which causal relationship is not clear. However, a standout point is that, by contrasting two groups of patients with CBP, our design allowed us to better isolate several clinical, psychosocial, and brain structural properties related to the prolonged use of opioids while controlling for clinical pain. Secondly, an important annotation has to be made regarding the clinical properties of both groups: opioid-treated patients presented with higher rates of previous back pain surgery, perhaps introducing confounding by indication. Although out of the scope of our study hypothesis, it is important to acknowledge two aspects: (1) lumbar surgery infrequently reduces the use of opioid therapy postoperatively if the patient was already on opioids; (2) among opioid-naive patients that undergo surgery there is a higher risk of initiating long-term opioids after surgery [79]. Although we did not inquire about the opioid prescription timing, we acknowledge that opioid-treated patients may have distinct prescription histories and their clinical pain presentation might, to some extent, relate to unsuccessful back surgery. However, our main goal was to control for patient-reported pain intensity and duration, which was achieved.

In conclusion, our multidimensional assessment including several instruments identified worse negative affect, patient-reported physical function, and worse pain interference associated with long-term opioid use. Comprehensive assessments including multidimensional measures may be helpful for clinical decision-making of continued opioid therapy. Furthermore, morphological brain imaging analysis identified brain alterations in the $\mathrm{dPaCC}$, independently of pain intensity and duration. Further studies are required to elucidate the clinical implications of the brain distortions induced by longterm opioid use. 

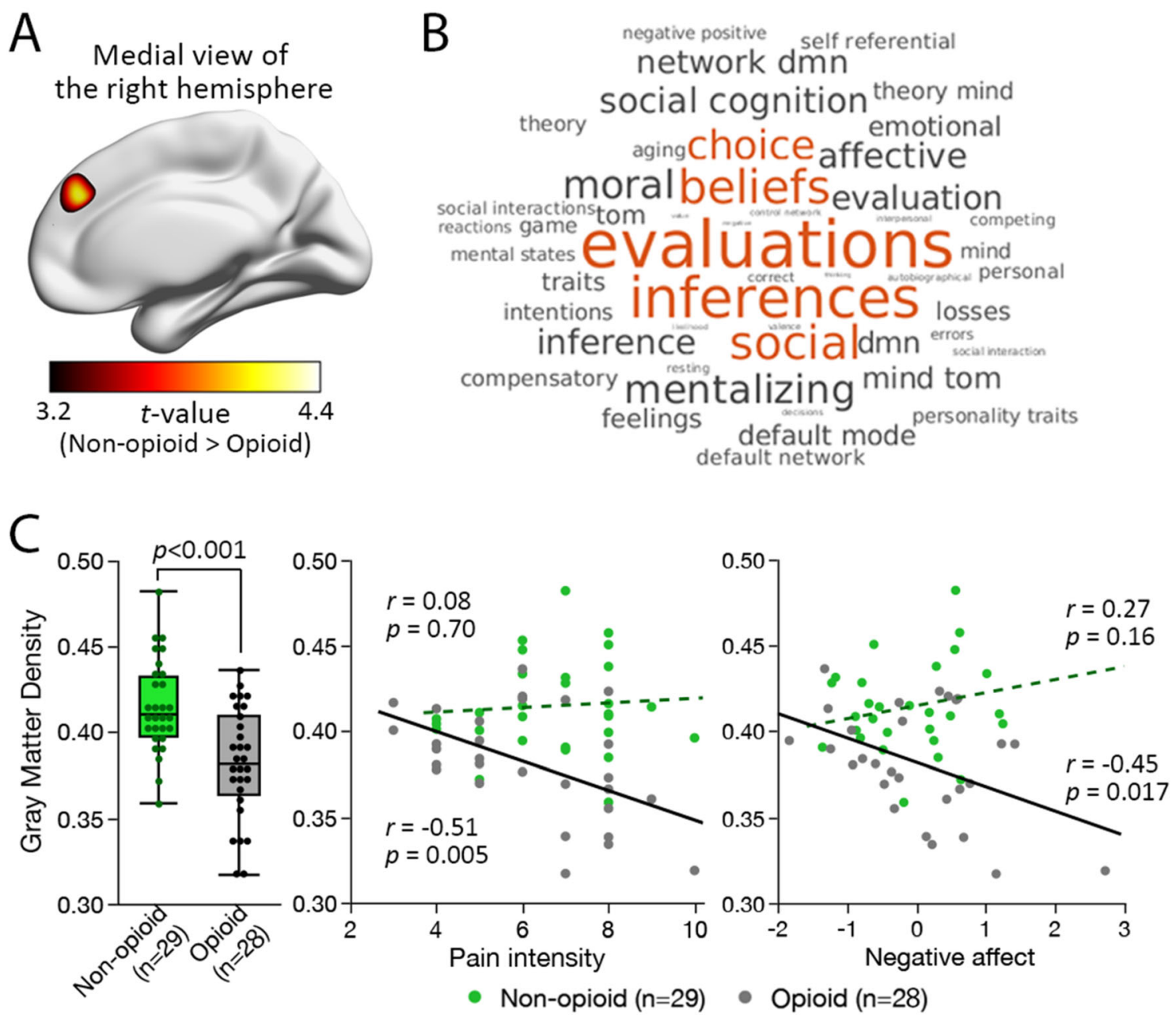

Fig. 3 Voxel-based morphometry between patients with chronic back pain without opioid $(n=29)$ and those with opioids $(n=29)$. a Patients with opioids showed decreased gray matter density of the dorsal paracingulate cortex ( $\mathrm{dPaCC})$. The voxel cluster including 538 voxels (center coordinate with the highest $t$ value was $x=+8 \mathrm{~mm}, y=+40 \mathrm{~mm}, z=+34 \mathrm{~mm}$ ) was identified by threshold-free cluster enhancement adjusted for age, sex, and intracranial volume (family-wise errorcollected $p$ value $<0.01$ ). b Word cloud of top 50 (of about 1700) terms associated with the identified cluster. The size of a term is in proportion to the correlation strength of each term to the brain region, generated by the Neurosynth reverse inference decoder. Orange color

\section{ACKNOWLEDGEMENTS}

We are grateful to all the participants in this study. represents top five correlated terms. c Group difference of the gray matter density and its associations with pain intensity and negative affect $(n=28)$. Opioid group showed statistically significant lower gray matter density in the $\mathrm{dPaCC}$ in a multivariable regression analysis adjusted for age, sex, history of back pain, pain intensity, pain duration, and intracranial volume. Both pain intensity and negative affect (principal component) were correlated with decreased gray matter density of the $\mathrm{dPaCC}$ in the patients with CBP with opioids, whereas there were no significant associations in patients with CBP not using opioids

Funding. This study was supported by the NIH Grant (1P50DA044121-01A1). This material is based upon work supported by the National Science Foundation Graduate Research 
Fellowship under Grant no. DGE-1324585. The journal's Rapid Service was funded by NIH NIDA P50 DA044121.

Authorship. All named authors meet the International Committee of Medical Journal Editors (ICMJE) criteria for authorship for this article, take responsibility for the integrity of the work as a whole, and have given their approval for this version to be published.

Authorship contributions. KW analyzed the data, and wrote a first draft of the manuscript. $\mathrm{ADV}, \mathrm{RJ}$ supported the data analyses. MA, JB, TJS participated in the data collection. AVA, MNB designed and conducted the study and supervised the data analysis. All authors revised the first draft for important intellectual content and contributed to the final manuscript.

Disclosures. Kenta Wakaizumi, Andrew D Vigotsky, Rami Jabakhanji, Maryam Abdallah, Joana Barroso, Thomas J Schnitzer, Apkar Vania Apkarian and Marwan N Baliki have nothing to disclose.

Compliance with ethics guidelines. This study was performed in accordance with the Helsinki Declaration of 1964 and its later amendments. All participants understood the purpose of the study, procedures to be completed, possible benefits and potential risks, and signed a consent form prior to any study-related activities. The protocols were approved by Northwestern University IRB (STU00207384, STU00205398).

Data availability. The data will be made available on OpenPain (www.openpain.org) once the full study and analysis are completed. Until then, data are available upon reasonable request.

Open Access. This article is licensed under a Creative Commons Attribution-NonCommercial 4.0 International License, which permits any non-commercial use, sharing, adaptation, distribution and reproduction in any medium or format, as long as you give appropriate credit to the original author(s) and the source, provide a link to the Creative Commons licence, and indicate if changes were made. The images or other third party material in this article are included in the article's Creative Commons licence, unless indicated otherwise in a credit line to the material. If material is not included in the article's Creative Commons licence and your intended use is not permitted by statutory regulation or exceeds the permitted use, you will need to obtain permission directly from the copyright holder. To view a copy of this licence, visit http://creativecommons.org/licenses/by$\mathrm{nc} / 4.0 /$.

\section{REFERENCES}

1. Treede RD, Rief W, Barke A, et al. Chronic pain as a symptom or a disease: the IASP Classification of Chronic Pain for the International Classification of Diseases (ICD-11). Pain. 2019;160(1):19-27. https:// doi.org/10.1097/j.pain.0000000000001384.

2. Dahlhamer J, Lucas J, Zelaya C, et al. Prevalence of chronic pain and high-impact chronic pain among adults-United States, 2016. MMWR Morb Mortal Wkly Rep. 2018;67(36):1001-6. https://doi.org/10. 15585/mmwr.mm6736a2.

3. Andrew R, Derry S, Taylor RS, Straube S, Phillips CJ. The costs and consequences of adequately managed chronic non-cancer pain and chronic neuropathic pain. Pain Pract. 2014;14(1):79-94. https://doi.org/ 10.1111/papr.12050.

4. Centers for Disease Control and Prevention. 2019 Annual surveillance report of drug-related risks and outcomes - United States Surveillance Special Report. Centers for Disease Control and Prevention, U.S. Department of Health and Human Services. 2019. https://www.cdc.gov/drugoverdose/pdf/ pubs/2019-cdc-drug-surveillance-report.pdf. Accessed 9 May 2020.

5. Spears CA, Hodges SE, Kiyani M, et al. Healthcare resource utilization and management of chronic, refractory low back pain in the United States. Spine. 2020 . 0000000000003572 . https://doi.org/10.1097/brs.

6. Hedegaard H, Miniño AM, Warner M. Drug overdose deaths in the United States, 1999-2018. NCHS Data Brief, no 356. Hyattsville, MD: National Center for Health Statistics. 2020.

7. Smith BC, Vigotsky AD, Apkarian AV, Schnitzer TJ. Temporal factors associated with opioid 
prescriptions for patients with pain conditions in an urban emergency department. JAMA Netw Open. 2020;3(3):e200802. https://doi.org/10.1001/ jamanetworkopen.2020.0802.

8. Dowell D, Haegerich TM, Chou R. CDC guideline for prescribing opioids for chronic pain-United States, 2016. JAMA. 2016;315(15):1624-45. https:// doi.org/10.1001/jama.2016.1464.

9. Edlund MJ, Martin BC, Russo JE, Devries A, Braden JB, Sullivan MD. The role of opioid prescription in incident opioid abuse and dependence among individuals with chronic non-cancer pain. Clin J Pain. 2013. https://doi.org/10.1097/ajp. 0000000000000021.

10. Chou R, Turner JA, Devine EB, et al. The effectiveness and risks of long-term opioid therapy for chronic pain: a systematic review for a national institutes of health pathways to prevention workshop. Ann Intern Med. 2015;162(4):276. https:// doi.org/10.7326/m14-2559.

11. Benyamin R, Trescot A, Datta S, et al. Opioid complications and side effects. Pain Physician. 2008;11(2Suppl):S105-20.

12. Solomon DH. The comparative safety of analgesics in older adults with arthritis. Arch Intern Med. 2010;170(22):1968. https://doi.org/10.1001/ archinternmed.2010.391.

13. Fishbain DA, Cutler R, Rosomoff HL, Rosomoff RS. Chronic pain-associated depression: antecedent or consequence of chronic pain? A review. Clin J Pain. 1997;13(2):116-37.

14. Solberg Nes L, Roach AR, Segerstrom SC. Executive functions, self-regulation, and chronic pain: a review. Ann Behav Med. 2009;37(2):173-83. https://doi.org/10.1007/s12160-009-9096-5.

15. Smith BH, Elliott AM, Chambers WA, Smith WC, Hannaford PC, Penny K. The impact of chronic pain in the community. Fam Pract. 2001;18(3): 292-9. https://doi.org/10.1093/fampra/18.3.292.

16. Gureje O, Von Korff M, Simon GE, Gater R. Persistent pain and well-being. JAMA. 1998;280(2):147. https://doi.org/10.1001/jama.280.2.147.

17. Mills SEE, Nicolson KP, Smith BH. Chronic pain: a review of its epidemiology and associated factors in population-based studies. $\mathrm{Br} \mathrm{J}$ Anaesth. 2019;123(2):e273-83. https://doi.org/10.1016/j.bja. 2019.03.023.

18. Lyoo IK, Pollack MH, Silveri MM, et al. Prefrontal and temporal gray matter density decreases in opiate dependence. Psychopharmacology.
2006;184(2):139-44. s00213-005-0198-x.

https://doi.org/10.1007/

19. Liu H, Hao Y, Kaneko Y, et al. Frontal and cingulate gray matter volume reduction in heroin dependence: optimized voxel-based morphometry. Psychiatry Clin Neurosci. 2009;63(4):563-8. https:// doi.org/10.1111/j.1440-1819.2009.01989.x.

20. Lin W-C, Chou K-H, Chen H-L, et al. Structural deficits in the emotion circuit and cerebellum are associated with depression, anxiety and cognitive dysfunction in methadone maintenance patients: a voxel-based morphometric study. Psychiatry Res Neuroimaging. 2012;201(2):89-97. https://doi.org/ 10.1016/j.pscychresns.2011.05.009.

21. Upadhyay J, Maleki N, Potter J, et al. Alterations in brain structure and functional connectivity in prescription opioid-dependent patients. Brain. 2010;133(7):2098-114. https://doi.org/10.1093/ brain/awq138.

22. Younger JW, Chu LF, D'Arcy NT, Trott KE, Jastrzab LE, Mackey SC. Prescription opioid analgesics rapidly change the human brain. Pain. 2011;152(8): 1803-10. https://doi.org/10.1016/j.pain.2011.03. 028.

23. Baliki MN, Schnitzer TJ, Bauer WR, Apkarian AV. Brain morphological signatures for chronic pain. PLoS One. 2011;6(10):e26010. https://doi.org/10. 1371/journal.pone.0026010.

24. Schmidt-Wilcke T, Hierlmeier S, Leinisch E. Altered regional brain morphology in patients with chronic facial pain. J Head Face Pain. 2010;50(8):1278-85. https://doi.org/10.1111/j.1526-4610.2010.01637.x.

25. Cauda F, Palermo S, Costa T, et al. Gray matter alterations in chronic pain: a network-oriented meta-analytic approach. NeuroImage Clin. 2014;4: 676-86. https://doi.org/10.1016/j.nicl.2014.04.007.

26. Farrar JT, Young JP Jr, LaMoreaux L, Werth JL, Poole RM. Clinical importance of changes in chronic pain intensity measured on an 11-point numerical pain rating scale. Pain. 2001;94(2):149-58.

27. Centers for Disease Control and Prevention. Analyzing prescription data and morphine milligram equivalents (MME). Centers for Disease Control and Prevention, National Center for Injury Prevention and Control. 2019. https://www.cdc.gov/ drugoverdose/resources/data.html. Accessed 9 May 2020.

28. Fairbank JC, Couper J, Davies JB, O'brien JP. The Oswestry low back pain disability questionnaire. Physiotherapy. 1980;66(8):271-3. 
29. Freynhagen R, Baron R, Gockel U, Tolle TR. painDETECT: a new screening questionnaire to identify neuropathic components in patients with back pain. Curr Med Res Opin. 2006;22(10):1911-20. https://doi.org/10.1185/030079906X132488.

30. Melzack R. The short-form McGill Pain Questionnaire. Pain. 1987;30(2):191-7.

31. Sullivan MJ, Bishop SR, Pivik J. The pain catastrophizing scale: development and validation. Psychol Assess. 1995;7(4):524.

32. Beck AT, Steer RA, Ball R, Ranieri W. Comparison of Beck Depression Inventories-IA and -II in psychiatric outpatients. J Pers Assess. 1996;67(3):588-97. https://doi.org/10.1207/s15327752jpa6703_13.

33. Watson D, Clark LA, Tellegen A. Development and validation of brief measures of positive and negative affect: the PANAS scales. J Pers Soc Psychol. 1988;54(6):1063-70.

34. Cella D, Choi SW, Condon DM, et al. PROMIS ${ }^{\circledR}$ adult health profiles: efficient short-form measures of seven health domains. Value Health. 2019;22(5): 537-44. https://doi.org/10.1016/j.jval.2019.02.004.

35. Ware JE, Kosinski M, Keller SD. A 12-item shortform health survey: construction of scales and preliminary tests of reliability and validity. Med Care. 1996;34(3):220-33.

36. Mehling WE, Price C, Daubenmier JJ, Acree M, Bartmess E, Stewart A. The Multidimensional Assessment of Interoceptive Awareness (MAIA). PLoS One. 2012;7(11):e48230. https://doi.org/10. 1371/journal.pone.0048230.

37. Hodes RJ, Insel TR, Landis SC. The NIH Toolbox: setting a standard for biomedical research. Neurology. 2013;80(11 Suppl):S1-S. https://doi.org/10. 1212/wnl.0b013e3182872e90.

38. Cook KF, Dunn W, Griffith JW, et al. Pain assessment using the NIH Toolbox. Neurology. 2013;80(11 Suppl):S49-53. https://doi.org/10.1212/ wnl.0b013e3182872e80.

39. Coldwell SE, Mennella JA, Duffy VB, Pelchat ML, Griffith JW, Smutzer G, et al. Gustation assessment using the NIH Toolbox. Neurology. 2013;80(11 Suppl):S20-4. 0b013e3182872e38.

40. Dalton P, Doty RL, Murphy C, et al. Olfactory assessment using the NIH Toolbox. Neurology. 2013;80(11 Suppl):S32-6. https://doi.org/10.1212/ wnl.0b013e3182872eb4.

41. Salsman JM, Butt Z, Pilkonis PA, et al. Emotion assessment using the NIH Toolbox. Neurology.
2013;80(11 Suppl):S76-86. https://doi.org/10.1212/ wnl.0b013e3182872e11.

42. Weintraub S, Dikmen SS, Heaton RK, et al. The cognition battery of the NIH Toolbox for assessment of neurological and behavioral function: validation in an adult sample. J Int Neuropsychol Soc. 2014;20(6):567-78. https://doi.org/10.1017/ s1355617714000320.

43. Reuben DB, Magasi S, McCreath HE, et al. Motor assessment using the NIH Toolbox. Neurology. 2013;80(11 Suppl):S65-75. https://doi.org/10.1212/ wnl.0b013e3182872e01.

44. Butler SF, Budman SH, Fernandez KC, et al. Development and validation of the current opioid misuse measure. Pain. 2007;130(1):144-56. https://doi.org/ 10.1016/j.pain.2007.01.014.

45. Shrive FM, Stuart H, Quan H, Ghali WA. Dealing with missing data in a multi-question depression scale: a comparison of imputation methods. BMC Med Res Methodol. 2006. https://doi.org/10.1186/ 1471-2288-6-57.

46. Smith SM, Zhang Y, Jenkinson M, et al. Accurate, robust, and automated longitudinal and cross-sectional brain change analysis. Neuroimage. 2002;17(1):479-89.

47. Smith SM, Jenkinson M, Woolrich MW, et al. Advances in functional and structural MR image analysis and implementation as FSL. Neuroimage. 2004;23:S208-19. neuroimage.2004.07.051.

48. Douaud G, Smith S, Jenkinson M, et al. Anatomically related grey and white matter abnormalities in adolescent-onset schizophrenia. Brain. 2007;130(9):2375-86. https://doi.org/10.1093/ brain/awm184.

49. Good CD, Johnsrude IS, Ashburner J, Henson RNA, Friston KJ, Frackowiak RSJ. A voxel-based morphometric study of ageing in 465 normal adult human brains. Neuroimage. 2001;14(1):21-36. https://doi. org/10.1006/nimg.2001.0786.

50. Smith S, Nichols T. Threshold-free cluster enhancement: addressing problems of smoothing, threshold dependence and localisation in cluster inference. Neuroimage. 2009;44(1):83-98. https:// doi.org/10.1016/j.neuroimage.2008.03.061.

51. Gorgolewski KJ, Varoquaux G, Rivera G, et al. NeuroVault.org: a repository for sharing unthresholded statistical maps, parcellations, and atlases of the human brain. Neuroimage. 2016;124:1242-4. https://doi.org/10.1016/j.neuroimage.2015.04.016. 
52. Yarkoni T, Poldrack RA, Nichols TE, Van Essen DC, Wager TD. Large-scale automated synthesis of human functional neuroimaging data. Nat Methods. 2011;8(8):665-70. https://doi.org/10.1038/ nmeth.1635.

53. Patenaude B, Smith SM, Kennedy DN, Jenkinson M. A Bayesian model of shape and appearance for subcortical brain segmentation. Neuroimage. 2011;56(3):907-22. https://doi.org/10.1016/j. neuroimage.2011.02.046.

54. Iglesias JE, Augustinack JC, Nguyen K, et al. A computational atlas of the hippocampal formation using ex vivo, ultra-high resolution MRI: application to adaptive segmentation of in vivo MRI. Neuroimage. 2015;115:117-37. https://doi.org/10. 1016/j.neuroimage.2015.04.042.

55. Xia M, Wang J, He Y. BrainNet Viewer: a network visualization tool for human brain connectomics. PLoS One. 2013;8(7):e68910. https://doi.org/10. 1371/journal.pone.0068910.

56. Burns JW, Bruehl S, France CR, et al. Psychosocial factors predict opioid analgesia through endogenous opioid function. Pain. 2017;158(3):391.

57. Nahman-Averbuch H, Nir RR, Sprecher E, Yarnitsky D. Psychological factors and conditioned pain modulation: a meta-analysis. Clin J Pain. 2016;32(6):541-54. https://doi.org/10.1097/AJP. 0000000000000296.

58. Scherrer JF, Salas J, Copeland LA, et al. Prescription opioid duration, dose, and increased risk of depression in 3 large patient populations. Ann Fam Med. 2016;14(1):54-62. https://doi.org/10.1370/ afm.1885.

59. Volkow ND, Wang GJ, Fowler JS, Tomasi D, Telang F. Addiction: beyond dopamine reward circuitry. Proc Natl Acad Sci. 2011;108(37):15037-42. https:// doi.org/10.1073/pnas.1010654108.

60. Heshmati M, Russo SJ. Anhedonia and the brain reward circuitry in depression. Curr Behav Neurosci Rep. 2015;2(3):146-53. https://doi.org/10.1007/ s40473-015-0044-3.

61. Dumas EO, Pollack GM. Opioid tolerance development: a pharmacokinetic/pharmacodynamic perspective. AAPS J. 2008;10(4):537-51. https://doi. org/10.1208/s12248-008-9056-1.

62. Sandhu H, Underwood M, Furlan AD, Noyes J, Eldabe S. What interventions are effective to taper opioids in patients with chronic pain? BMJ. 2018;362:k2990. https://doi.org/10.1136/bmj. k2990.
63. Bair MJ, Robinson RL, Katon W, Kroenke K. Depression and pain comorbidity: a literature review. Arch Intern Med. 2003;163(20):2433-45. https://doi.org/10.1001/archinte.163.20.2433.

64. Goodwin RD. Association between physical activity and mental disorders among adults in the United States. Prev Med. 2003;36(6):698-703. https://doi. org/10.1016/S0091-7435(03)00042-2.

65. Ashworth J, Green DJ, Dunn KM, Jordan KP. Opioid use among low back pain patients in primary care: is opioid prescription associated with disability at 6-month follow-up? Pain. 2013;154(7):1038-44. https://doi.org/10.1016/j.pain.2013.03.011.

66. Vogt BA, Nimchinsky EA, Vogt LJ, Hof PR. Human cingulate cortex: surface features, flat maps, and cytoarchitecture. J Comp Neurol. 1995;359(3): 490-506. https://doi.org/10.1002/cne.903590310.

67. Bush G, Vogt BA, Holmes J, et al. Dorsal anterior cingulate cortex: a role in reward-based decision making. Proc Natl Acad Sci. 2002;99(1):523-8. https://doi.org/10.1073/pnas.012470999.

68. Ridderinkhof KR. The role of the medial frontal cortex in cognitive control. Science. 2004;306(5695):443-7. https://doi.org/10.1126/ science. 1100301.

69. Hirvonen J, Aalto S, Hagelberg N, et al. Measurement of central $\mu$-opioid receptor binding in vivo with PET and [11C]carfentanil: a test-retest study in healthy subjects. Eur J Nucl Med Mol Imaging. 2009;36(2):275-86. https://doi.org/10.1007/ s00259-008-0935-6.

70. Leppä M, Korvenoja A, Carlson S, et al. Acute opioid effects on human brain as revealed by functional magnetic resonance imaging. Neuroimage. 2006;31(2):661-9. https://doi.org/10.1016/j. neuroimage.2005.12.019.

71. Bora E, Fornito A, Pantelis C, Yücel M. Gray matter abnormalities in major depressive disorder: a metaanalysis of voxel based morphometry studies. J Affect Disord. 2012;138(1-2):9-18. https://doi.org/10. 1016/j.jad.2011.03.049.

72. Du M-Y, Wu Q-Z, Yue Q, et al. Voxelwise metaanalysis of gray matter reduction in major depressive disorder. Prog Neuropsychopharmacol Biol Psychiatry. 2012;36(1):11-6. https://doi.org/10. 1016/j.pnpbp.2011.09.014.

73. Rodriguez-Raecke R, Niemeier A, Ihle K, Ruether W, May A. Brain gray matter decrease in chronic pain is the consequence and not the cause of pain. J Neurosci. 2009;29(44):13746-50. https://doi.org/10. 1523/jneurosci.3687-09.2009. 
74. Wollman SC, Alhassoon OM, Hall MG, et al. Gray matter abnormalities in opioid-dependent patients: a neuroimaging meta-analysis. Am J Drug Alcohol Abuse. 2017;43(5):505-17. https://doi.org/10.1080/ 00952990.2016 .1245312 .

75. Arteta J, Cobos B, Hu Y, Jordan K, Howard K. Evaluation of how depression and anxiety mediate the relationship between pain catastrophizing and prescription opioid misuse in a chronic pain population. Pain Med. 2016;17:295-303. https://doi. org/10.1111/pme.12886.

76. Hah J, Sturgeon J, Zocca J, Sharifzadeh Y, Mackey S. Factors associated with prescription opioid misuse in a cross-sectional cohort of patients with chronic non-cancer pain. J Pain Res. 2017;10:979-87. https://doi.org/10.2147/jpr.s131979.
77. Webster LR, Markman J, Cone EJ, Niebler G. Current and future development of extended-release, abuse-deterrent opioid formulations in the United States. Postgrad Med. 2017;129(1):102-10. https:// doi.org/10.1080/00325481.2017.1268902.

78. Stanos S. Evolution of opioid risk management and review of the classwide REMS for extended-release/long-acting opioids. Phys Sportsmed. 2012;40(4):12-20. https://doi.org/10.3810/psm. 2012.11.1975.

79. Deyo RA, Hallvik SE, Hildebran C, et al. Use of prescription opioids before and after an operation for chronic pain (lumbar fusion surgery). Pain. 2018;159(6):1147. 